\title{
Phase Magnitude Diagram for Linear Systems with Delay
}

\author{
Santiago Garrido, Fernando Martín, Luis Moreno \\ Universidad Carlos III de Madrid, sgarrido@ing.uc3m.es
}

\begin{abstract}
Transfer functions can be viewed as complex functions where the magnitude and the phase of a system depend on complex numbers. In our previous work, we have developed a graphical tool where transfer functions are represented in a fourdimensional space. The magnitude is represented in decibels in the third dimension and a color code is applied to represent the phase in a fourth dimension. Level curves are used to compare points with the same magnitude or phase. This tool, which is called Phase Magnitude diagram, makes it more intuitive to understand complex functions. This representation allows the user to visually obtain the phase and the gain that have to be added to a system to meet some design specifications. After that, any control approach can be employed. The application to systems with delay is discussed in this paper. It is demonstrated that the Phase Magnitude diagram can be efficiently applied to design an ideal PD controller for a system with delay. In addition, it is deduced that this instrument is not limited to this class of system.
\end{abstract}

Keywords: Transfer Function, Phase Magnitude diagram, Linear systems with delay, System with Delay, Control Engineering

\section{INTRODUCTION}

Most typical diagrams used in Control Engineering, such as Root Locus, Bode, Nichols, or Nyquist, show partial aspects of the Transfer Function (TF). In all of them, it is possible to extract some characteristics of the system and to design different types of controllers to improve the system behavior. Nowadays, with tools such as MATLAB SISOTOOL, it is viable to see many of these diagrams at the same time and the time response of the system to tune the controller. However, they are not a complete representation of the TF. Besides, the basic version of these diagrams is only useful when the system to be controlled presents a linear structure.

TFs are complex functions, $G(s): \mathbb{C} \longrightarrow \mathbb{C}$, and
$\mathbb{C}$ is represented by two variables. Therefore, it is necessary to have 4 axes to represent $G(s)$. Our brain is trained to visualize objects in three spatial dimensions, while the graphs of complex functions live in a four-dimensional space.

Complex functions are difficult to represent and to study. In some papers $[1,2,3]$ and books $[4,5,6]$ about complex variables, the researchers have represented complex functions in many ways that include colors to incorporate the phase. In general, the problem of these approaches is that the representations do not permit to obtain accurate measurements.

In our previous work, we have developed a tool that is easy to implement and contains all the information of the TF [7]. This tool has been called Phase Magnitude (PM) diagram. Its main characteristic is that the magnitude and the phase of the TF can be visually identified in a simple graph. This tool was intuitively applied to design multiple different controllers for linear systems. The method relies on the classic control approaches (Root Locus, Bode, etc), but the starting point is a visual identification of the magnitud and the phase that have to be added by the controller in order to meet the design specifications, which makes this technique a very intuitive method for the engineer. In addition, the graphical representation allows us to understand the behavior of the function, which is more difficult if only the equation is available.

As will be demonstrated in this paper, this tool could be specially useful when applied to nonlinear systems. Instead of applying complicated techniques which are characteristic of nonlinear processes, the same simple ideas applied to design the PM-based controller for the linear system can be followed. This paper covers the application of this method to design controllers for linear systems with a constant delay. The algorithm can also be applied to any nonlinear system if it can be modeled by a $\mathrm{TF}$.

The rest of this paper is organized as follows. The main concepts of the PM diagram are explained in Section 2. After that, Section 3 details the 
application for linear systems with delay. The experiments that validate our method to design controllers are shown in Section 4 and, finally, the most important conclusions are summarized in Section 5 .

\section{PHASE MAGNITUDE DIAGRAM}

In the PM diagram, the variables that completely define the TF will be represented: two of them correspond to the complex variables in the $s$ plane and the other two are the magnitude and the phase of the TF for the given $s$.

The $s$-plane will be represented by two axes, following the same notation that is used in other classic graphical tools such as the Root Locus.

In order to completely depict the $\mathrm{TF}$, the solution adopted by the PM approach uses level curves in a third dimension to identify the system magnitude. The function magnitude is represented in decibels, $|G(s)|_{d B}=20 \log |G(s)|$, and level curves in a third dimension are utilized to compare points in the $s$-plane that share the same value for the magnitude. This variable can be plotted in a three-dimensional graph or in a projection in a two-dimensional plane. The magnitude level curves are equally spaced in the diagram with increments of $2 d B$. As can be deduced, the difference between two consecutive lines is the same in decibels.

The phase of the TF, which is the fourth variable to be added to the PM diagram, is identified by colors. Color-coded values in the domain of the function are applied to show the phase of the $\mathrm{TF}$ for a given value in the $s$-plane. Therefore, another set of curves is used for $\arg [G(s)]$. The scale is linear, with red representing $0^{\circ}$ and cyan for $180^{\circ}$. This means that the cyan line is the Root Locus and the red line is the Inverse Root Locus. The phases are also equally spaced with increments of $20^{\circ}$.

A simple example of the PM diagram for $G(s)=$ $1 /(s+1)(s+3)$ can be observed in Figures 1 and 2 . The three-dimensional view is shown in Figure 1. The $s$-plane is represented in the horizontal axes. The vertical axis and the corresponding level curves show the different magnitudes. The reader can check that the magnitude tends towards the infinity when $s$ is equal to -1 or -3 , which are the poles of the open-loop system. Each point in the complex plane has an associated color.

The plane projection of the PM diagram is displayed in Figure 2. This is the form of the PM diagram that is more useful when designing con-

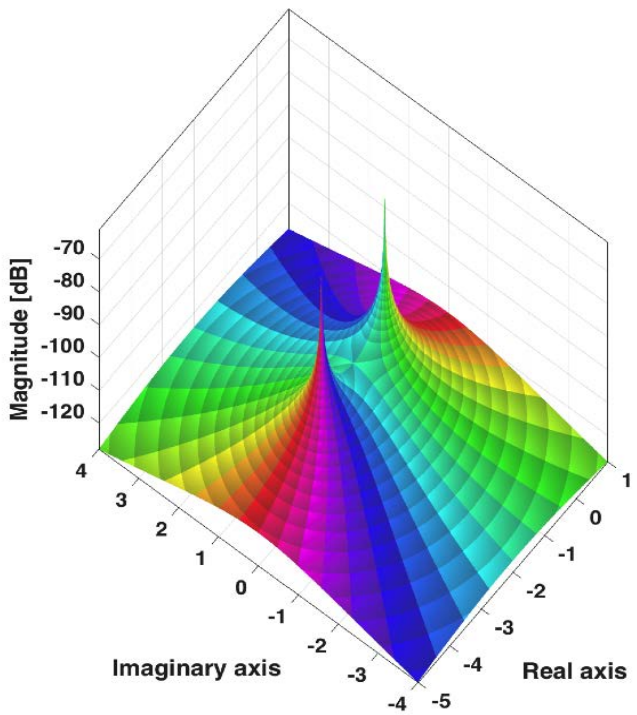

Figure 1: PM diagram for $G(s)=1 /(s+1)(s+3)$. Three-dimensional view.

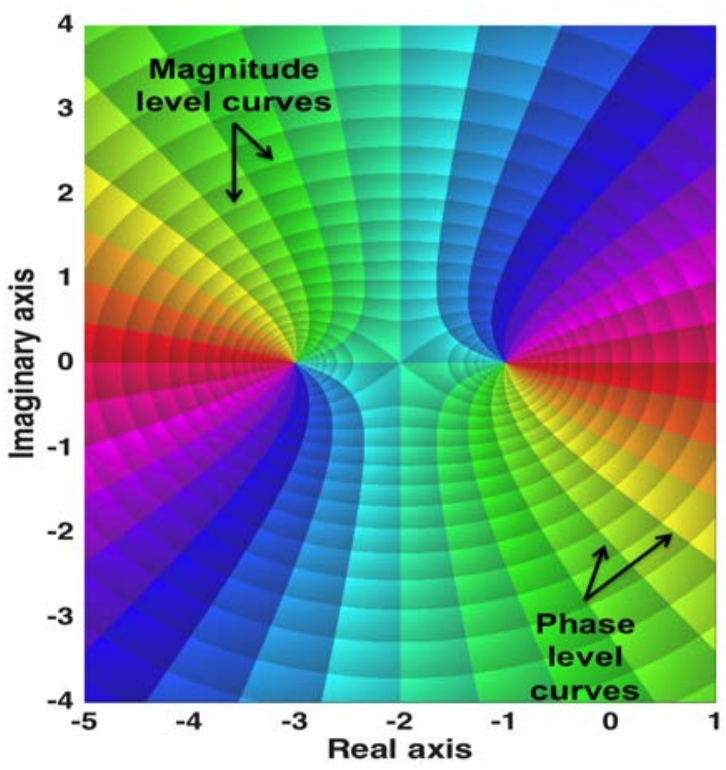

Figure 2: PM diagram for $G(s)=1 /(s+1)(s+3)$. Planar view.

trollers. This is mainly because it is very intuitive to read the magnitude and the phase that are needed to obtain poles in closed-loop in a desired positions.

For example, imagine that the closed-loop poles of the actual system (without controller) are situated in $-1.5 \pm 1.5 j$. The requirements are to obtain closed-loop poles in $-2.5 \pm 2.5 j$. The number of lines in the phase reference frame between $-1.5 \pm$ 1.5 and $-2.5 \pm 2.5$ correspond to the phase that must be added by the controller $\left(P_{d i f-P M}\right)$. The same happens with the magnitude level curves, 
and the magnitude difference is called $M_{d i f-P M}$.

In the classical control approach based on the Root Locus diagram, the Argument Condition is applied to compute how the Root Locus has to be modified to contain the dominant poles. After that, the Magnitude Condition is applied to have the closed-loop poles in the desired positions. In other words, the same variables that can be intuitively obtained using the PM diagram are computed and, after that, taken into account to meet the specifications.

Therefore, the main difference is that, instead of following tedious calculation methods, the values can be easily obtained from the graph. After that, any control technique could be applied. For example, after reading the phase, the controller $C_{1}(s)$ with gain one that adds that phase $\left(P_{\text {dif }-P M}\right)$ can be calculated. Then, the PM diagram of $C_{1}(s) G(s)$ is represented and, finally, the gain that is needed $\left(M_{d i f-P M}\right)$ is used to compute the final expression of the controller $C(s)$.

The PM approach has many other advantages. It permits to study the time domain and the frequency domain responses at the same time. It is also possible to put a grid over the PM diagram in order to read values of other variables such as the damping ratio $\zeta$ or the natural frequency $\omega_{n}$. It allows to read the Phase Margin directly because it is the phase distance from the actual closedloop poles position following the same magnitude line until the intersection with the imaginary axis. The Gain Margin is the magnitude distance following the cyan line that represents $180^{\circ}$ until its intersection with the imaginary axis. The imaginary axis cut represents the Bode diagram. In the drawings, linear scales have been used for the real and imaginary axes, but if there are poles and zeros placed in different decades, it would be better to put log scales. Another advantage, which will be exploited in this work, is that any kind of complex function could be represented, thus it is possible to represent linear and nonlinear systems.

This representation can be considered as a Root Contour diagram with two parameters: gain and phase $[8,9]$. It is not the first time that level curves are used to represent magnitude and phase of a function. A related approach can be found in the work of Cavicchi [6]. The main drawback of his approach is the bad resolution and the difficulty of reading.

This technique was successfully applied in our previous work to design different controllers for linear systems. The objective of this paper is to expand our study to different systems, starting with systems with delay, which are explained in the next section.

\section{SYSTEMS WITH DELAY}

The study of linear systems is a traditional topic in control engineering. Among them, systems with delay are a very common class of systems that are defined by the following equation:

$$
G_{D}(s)=G(s) e^{-T s},
$$

where $T$ is the system delay and it is assumed that $G(s)$ is a linear TF.

It is not straightforward to deduce the control method that could be applicable to these systems. If a classical control approach that does not take into account the delay is applied, the system could be unstable, and the control requirements would not be satisfied. Besides, classical graphical tools such as the Root Locus method cannot be directly applied when the TF includes the exponential term $e^{-T s}$.

A typical method to control these systems is based on the Smith's Predictor (SP) [10]. The SP is a design method for systems with delay that relies on a change in the structure of the controller. First, a controller is computed without taking into account the delay. After that, the structure of the controller is modified with the objective of obtaining a closed-loop system where the delay is situated outside the control loop. In this way, the equivalent system will be delayed, but the delay is outside the control loop and the system will be stable. Mathematical equivalences are used to calculate the architecture of the new controller.

However, since it is a complex function, it is possible to calculate the magnitude and the phase of $G_{D}(s)$ following the same ideas described in Section 2. Therefore, the PM diagram could be an interesting technique to design adequate controllers for this type of system.

In addition, if other nonlinear systems can be defined by a complex TF, the PM diagram is not limited to systems with delay, and the application of this technique to design controllers for other nonlinear models can be viewed as a challenging work to be accomplished as future research. For instance, the study of fractional-order systems is a promising field of application, since these systems can be modeled by a TF and other authors have already applied techniques such us the Root Locus method to represent the fractional roots [11].

An example will be shown to illustrate how the PM diagram can be easily computed for a system with delay. The PM of the following TF is repre- 


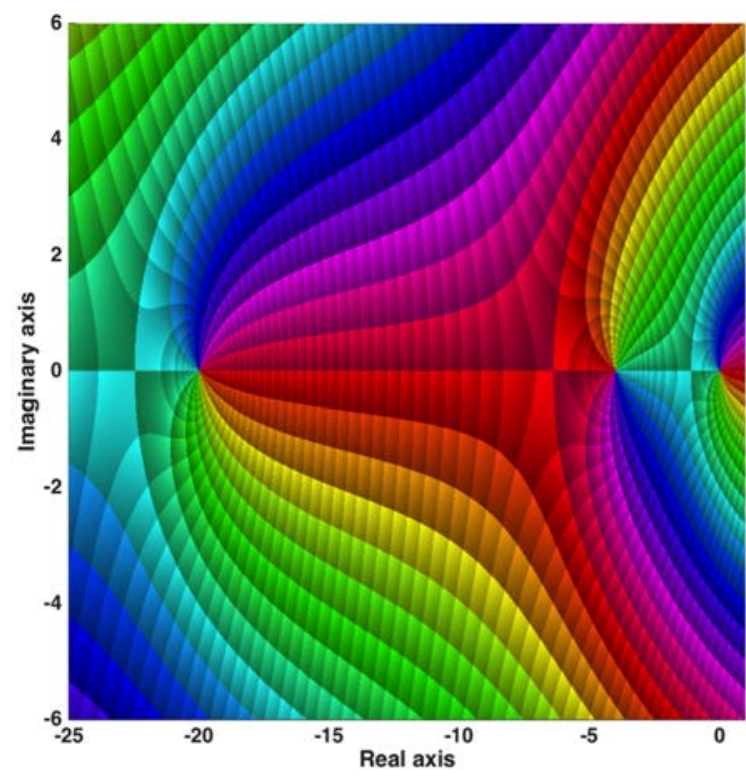

Figure 3: PM diagram for a system with delay.

sented in Figure 3:

$$
G_{D}=\frac{300}{(s+20)(s+4) s} e^{-0.5 s} .
$$

It can be observed that, when compared to a linear system without delay, the term $e^{-T s}$ displaces the system to the positive real plane, increasing the zone of gains (if the Root Locus is analyzed) where the system is unstable.

Since the exact values of the complex TF are represented in the PM diagram, this method can be used to visually obtain the gain and phase that is necessary to add to the system to meet any design specifications. An example with an ideal PD controller is shown in the next section.

\section{EXPERIMENTS}

In this section, a simple ideal PD controller is designed using the PM diagram. The starting point is the PM diagram of a system with delay displayed in Figure 3. Two parameters are needed to compute the magnitude and the phase that have to be added by the controller. First, the operation mode of the system (magnitude and phase of $G_{D}(s)$ in a closed-loop system without controller). These values correspond to the poles of the Root Locus when the gain is one, and they can be identified in the PM diagram. Second, the dominant poles that make the system meet the specifications.

To illustrate these concepts, Figure 4 shows an enlarged version of Figure 3 where the aforementioned values are marked with dots.

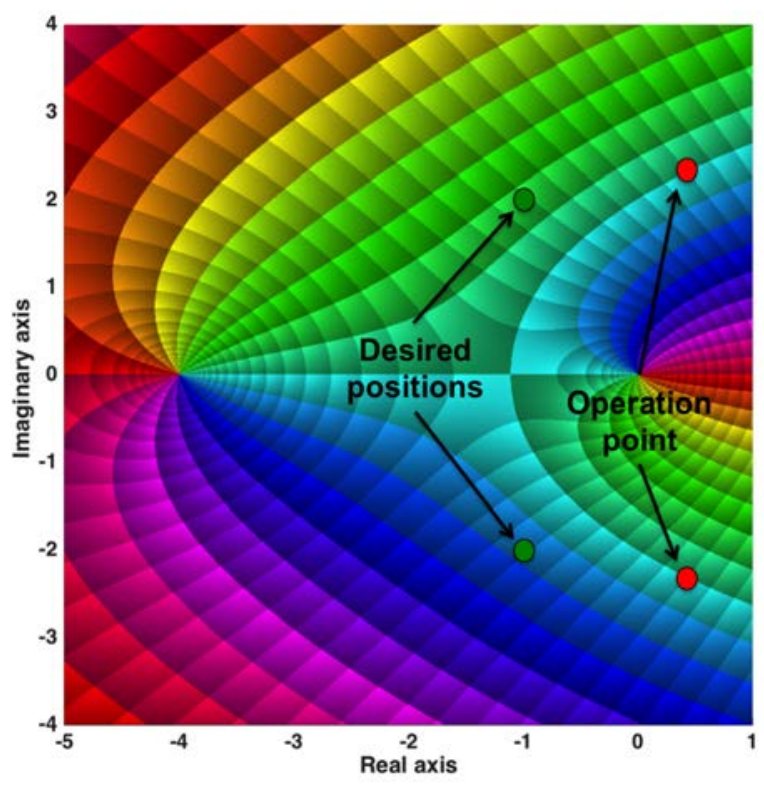

Figure 4: Zoom of PM diagram for a system with delay.

Red dots are used to show the operation point of the system without controller. As said before, these poles can be easily calculated because they correspond to the values of the Root Locus when $G_{D}(s)$ is in a closed-loop system without controller. Green points mark the desired poles to meet the specifications. In this case, the objective is to obtain poles in closed loop are equal to $-1 \pm 2 j$. These poles mean that the stabilization time would be approximately equal to 3.14 $\mathrm{s}$ and the overshoot would be $20.79 \%$ if the classical assumptions to calculate this parameters in second-order systems were considered. However, the system is not a classic linear second-order system. In fact, a delay is included, thus the objective poles do not correspond to the cited requirements.

This system has the closed-loop poles placed in $0.429 \pm 2.317 j$. The magnitude of $G_{D}$ in the dominant poles is $\left|G_{D}(0.429 \pm 2.317 j)\right|=1(0$ $\mathrm{dB})$ and the phase is $\arg \left(G_{D}(0.429 \pm 2.317 j)\right)=$ $180^{\circ}$. The desired specifications give us that the desired closed loop poles have to be placed in $-1 \pm 2 j$. The magnitude and phase of $G_{D}(s)$ in these points are $\left|G_{D}(-1 \pm 2 j)\right|=3.2(10.13 \mathrm{~dB})$ and $\arg \left(G_{D}(-1 \pm 2 j)\right)=34.61^{\circ}$.

The next step is to compute the phase and the magnitude that are needed to meet the requirements. These values can be visually and intuitively obtained from the diagram. Observing the figure, there are 1.7 (between one and two) phase level lines between the red and the green dot, thus $34^{\circ}$ are required. Analyzing the magnitude level curves, both dots are separated by approximately 5 lines. Since one line corresponds to two $d B, 10$ 


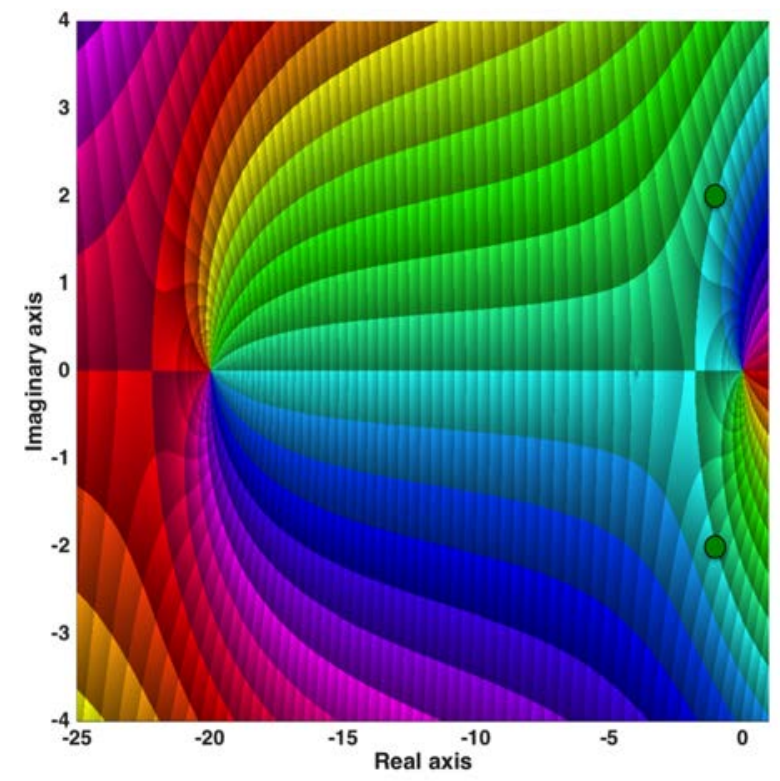

Figure 5: PM diagram for $G_{D}(s)$ with the controller included.

$d B$ have to be added. The magnitude level curves can be studied from another perspective. It is also true to say that the gain has to be multiplied by 3.2 when the controller is included. Both ideas are valid and equivalent when designing a controller.

Once the phase and the gain to be added are obtained from the PM diagram, any control method could be applied to meet the requirements. In this case, the example is illustrated by the obtention of a simple ideal PD controller. The Root Locus method is applied to obtain an ideal PD controller that modifies the system to meet the requirements according to the phase and gain to be added. The controller is calculated by a MATLAB script and the new PM diagram is displayed. The controller $C(s)$ that makes the system meet the specifications is

$$
C(s)=0.0845(s+4.3912) \text {. }
$$

Figure 5 shows the PM diagram of the controlled system. It can be observed that the desired poles are now included in the Root Locus (green dots in the figure). Therefore, the controller is adequate to meet the design specifications. The step response of the system is detailed in Figure 6. The system output is delayed 0.5 seconds, but it is stable and it meets the design requirements.

The stabilization time is approximately $3 \mathrm{~s}$ (including the delay) and the peak time is $2 \mathrm{~s}$. The system overshoot is $20 \%$ and the steady-state error is $0 \%$, which is logical because the plant $\mathrm{TF}$ contains an integrator.

As can be observed in the figure, the inclusion
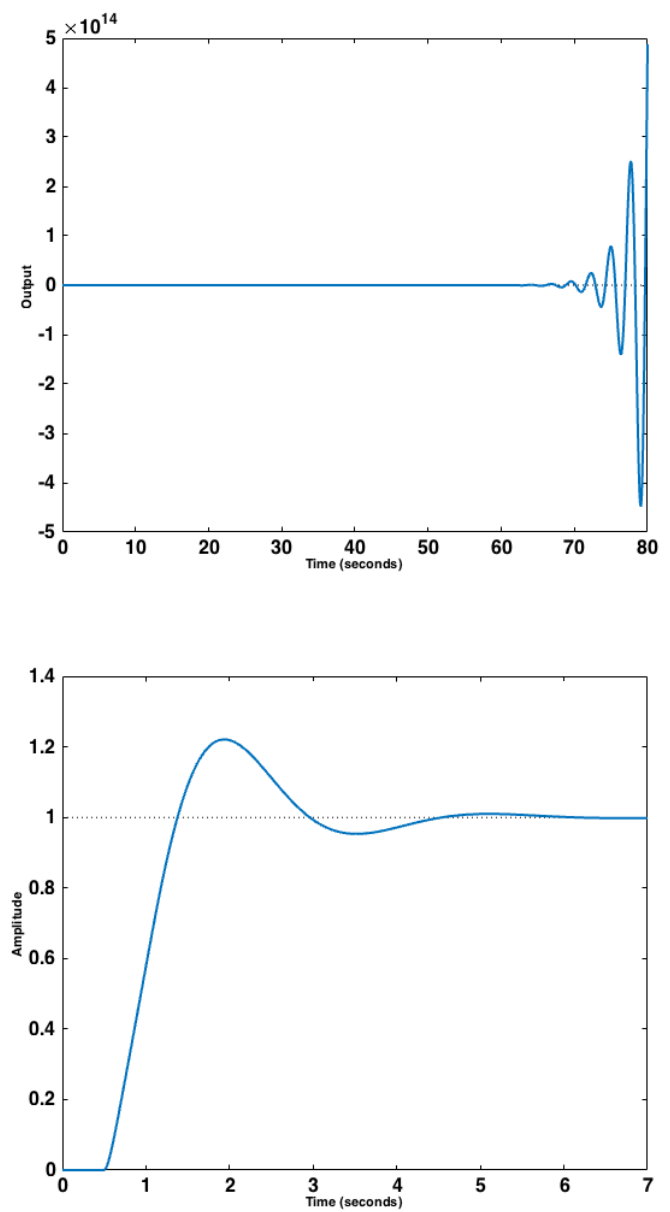

Figure 6: Top: step response of the system without controller in the operation point. Bottom: step response of the system with controller.

of the delay makes the system unstable. When the PD controller is added with the values given by the PM diagram, the system output is stable, obtaining a signal similar to those one obtained when a specific technique for systems with delay such as the SP is applied.

\section{CONCLUSIONS}

In this work, the PM diagram has been applied to demonstrate that this technique can be used to design controllers for linear systems with a constant delay. Since any complex function can be represented using this diagram, it is a suitable method for this type of systems when the nonlinear model can be defined by a complex function (like the $\mathrm{TF})$.

One of the main advantages is that a simple graphical tool can be applied to nonlinear systems, instead of the complicated control approaches that are characteristic of these systems. If the sys- 
tem can be represented by the PM diagram, it is straightforward to read the phase and the magnitude that are necessary to add to satisfy some design requirements. The intuitive and visual obtention of these parameters is another advantage to mention because, among other things, it makes it easier to understand the behavior of complex systems. After that, any control method could be applied to obtain the controller that produces the desired changes in magitude and phase.

In particular, the PM diagram has been applied to model a linear system with delay. It is a useful tool for these systems because they can be modeled by a TF. An ideal PD controller is tuned for the system with delay following the Root Locus design method. The good behavior of the controlled system can be appreciated in the experiments.

The next step to be considered as future research is to apply these ideas to different systems. Nonlinear systems have to be studied to check if they can be modeled by the graphical tool. Fractional systems are a promising field to apply our method because they rely on a TF representation.

\section{References}

[1] Wegert, E., Semmler, G., Phase plots of complex functions: a journey in illustration. Notices AMS vol. 58. (768-780). 2010.

[2] Wegert, E., Visual Complex Functions: An Introduction With Phase Portraits. Birkhuser. 2012

[3] Needham, T., Visual Complex Analysis. Clarendon Press. 1998

[4] Poelke, K., Polthier, K., Lifted domain coloring. Computer Graphics Forum. Vol. 28. No. 3. Blackwell Publishing Ltd, 2009.

[5] Mathews, J., R. Howell , R., Complex Analysis for Mathematics and Engineering. Jones and Bartlett Learning. 2010.

[6] Cavicchi, T.J., Phase-Root Locus and Relative Stability. IEEE Contr. Syst. Mag., vol 16, pp. 69-76, 1996.

[7] Garrido, S., Moreno, L. PM Diagram of the Transfer Function and Its Use in the Design of Controllers. Journal of Mathematics and System Science, 5, 138-149, 2015

[8] Ogata, K., Modern Control Engineering, 5th. ed. Prentice Hall, 2009.

[9] Kuo, B. C., Automatic Control Systems, 6th ed.. Prentice Hall, 1991.
[10] Astrom, K. J., Hang, C. C., Lim, B. C. A new Smith predictor for controlling a process with an integrator and long dead-time. IEEE transactions on Automatic Control, 39(2), 343-345, 1994.

[11] Tenreiro Machado, J. A., Root locus of fractional linear systems. Commun Nonlinear Sci Numer Simulat vol 16, pp. 3855-3862, 2011. 